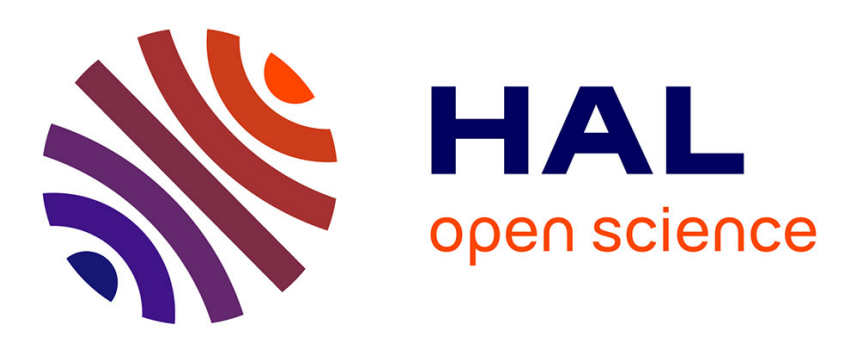

\title{
A Shape-based Statistical Method to Retrieve 2D TRUS-MR Slice Correspondence for Prostate Biopsy
}

Jhimli Mitra, Abhilash Srikantha, Désiré Sidibé, Robert Marti, Arnau Oliver, Xavier Llado, Soumya Ghose, Joan C. Vilanova, Josep Comet, Fabrice Mériaudeau

\section{To cite this version:}

Jhimli Mitra, Abhilash Srikantha, Désiré Sidibé, Robert Marti, Arnau Oliver, et al.. A Shape-based Statistical Method to Retrieve 2D TRUS-MR Slice Correspondence for Prostate Biopsy. SPIE Medical Imaging, Feb 2012, San Diego, United States. pp.1-9. hal-00658088

\section{HAL Id: hal-00658088 \\ https://u-bourgogne.hal.science/hal-00658088}

Submitted on 9 Mar 2012

HAL is a multi-disciplinary open access archive for the deposit and dissemination of scientific research documents, whether they are published or not. The documents may come from teaching and research institutions in France or abroad, or from public or private research centers.
L'archive ouverte pluridisciplinaire HAL, est destinée au dépôt et à la diffusion de documents scientifiques de niveau recherche, publiés ou non, émanant des établissements d'enseignement et de recherche français ou étrangers, des laboratoires publics ou privés. 


\title{
A shape-based statistical method to retrieve 2D TRUS-MR slice correspondence for prostate biopsy
}

\author{
Jhimli Mitra ${ }^{a, b}$, Abhilash Srikantha $^{a}$, Désiré Sidibé ${ }^{a}$, Robert Martí $^{b}$, Arnau Oliver $^{b}$ \\ Xavier Lladó ${ }^{b}$, Soumya Ghose ${ }^{a, b}$, Joan C. Vilanova ${ }^{c}$, Josep Comet ${ }^{d}$ and Fabrice Meriaudeau ${ }^{a}$ \\ ${ }^{a}$ Université de Bourgogne, Le2i-UMR CNRS 5158, Le Creusot, France. \\ ${ }^{b}$ Universitat de Girona, Computer Vision and Robotics Group, Girona, Spain. \\ ${ }^{c}$ Girona Magnetic Resonance Center, Girona, Spain. \\ ${ }^{d}$ Hospital Dr. Josep Trueta, Girona, Spain.
}

\begin{abstract}
This paper presents a method based on shape-context and statistical measures to match interventional 2D Trans Rectal Ultrasound (TRUS) slice during prostate biopsy to a 2D Magnetic Resonance (MR) slice of a pre-acquired prostate volume. Accurate biopsy tissue sampling requires translation of the MR slice information on the TRUS guided biopsy slice. However, this translation or fusion requires the knowledge of the spatial position of the TRUS slice and this is only possible with the use of an electro-magnetic (EM) tracker attached to the TRUS probe. Since, the use of EM tracker is not common in clinical practice and 3D TRUS is not used during biopsy, we propose to perform an analysis based on shape and information theory to reach close enough to the actual MR slice as validated by experts. The Bhattacharyya distance is used to find point correspondences between shape-context representations of the prostate contours. Thereafter, Chi-square distance is used to find out those MR slices where the prostates closely match with that of the TRUS slice. Normalized Mutual Information (NMI) values of the TRUS slice with each of the axial MR slices are computed after rigid alignment and consecutively a strategic elimination based on a set of rules between the Chi-square distances and the NMI leads to the required MR slice. We validated our method for TRUS axial slices of 15 patients, of which 11 results matched at least one experts validation and the remaining 4 are at most one slice away from the expert validations.
\end{abstract}

Keywords: Prostate biopsy, 2D TRUS/3D MR correspondence, shape-context, Chi-square distance, normalized mutual information.

\section{INTRODUCTION}

Prostate cancer has been a major cause of mortality among human males in the European and American societies since the last 25 years. Therefore, prostate cancer screening programs are conducted where a patient with abnormal findings after a digital rectal examination, serum Prostate Specific Antigen (PSA) level over 4.0ng/ml and PSA velocity between 0.4 to $0.75 \mathrm{ng} / \mathrm{ml} / \mathrm{yr}$ is generally advised with a prostate biopsy for histopathological examination of the prostate tissues. The appearance of malignant lesions in a Transrectal Ultrasound (TRUS) guided needle biopsy is mostly hypoechoic and the accuracy of finding such lesions is typically $43 \%$ in sonography. ${ }^{1}$ Approximately $25 \%$ - $42 \%$ of cancer lesions can also be isoechoic under TRUS. Therefore, the probability to diagnose malignant tissue from TRUS multi-core biopsy alone is $70 \%-80 \%{ }^{2}$ Vilanova et al. ${ }^{3}$ demonstrated

Further author information: (Send correspondence to Jhimli Mitra or Dr. Robert Martí or Prof. Fabrice Meriaudeau.) Jhimli Mitra: E-mail: jhimlimitra@yahoo.com,

Abhilash Srikantha: E-mail: aalibash@gmail.com,

Désiré Sidibé: E-mail: dro-desire.sidibe@u-bourgogne.fr,

Robert Martí: E-mail: marly@eia.udg.edu, Telephone: +34972418876

Arnau Oliver: E-mail: aoliver@eia.udg.edu,

Xavier Lladó: E-mail: llado@eia.udg.edu,

Soumya Ghose: E-mail: soumyaghose@gmail.com,

Fabrice Meriaudeau: E-mail: fabrice.meriaudeau@u-bourgogne.fr, Telephone: +33(0)385731077 
that the accuracy of Magnetic Resonance Imaging (MRI) to diagnose prostate cancer is $95 \%$ and detection of prostate cancer using MRI has a negative predictive value greater than $95 \%$. Therefore, MRI may serve as a triage for men deemed to be at risk of prostate cancer. In other words, if MRI has a negative predictive value greater than $95 \%$ for significant cancer then it might greatly reduce the number requiring biopsy, while at the same time increasing the yield of useful information for those who are sent for biopsy. Therefore, fusion of pre-biopsy MR images onto intraoperative TRUS images might increase the overall biopsy accuracy. ${ }^{4}$

Registration of pre-biopsy MR with intraoperative TRUS may be done in several ways. An Electro Magnetic (EM) tracker attached to the 2D TRUS probe may be used that sweeps the prostate to reconstruct a 3D TRUS volume. The 3D TRUS volume is then fused with the MR volume to obtain the spatial position of the $2 \mathrm{D}$ TRUS slice during biopsy within the pre-biopsy MR volume. ${ }^{4}$ On the other hand, a 3D TRUS probe may be directly used to acquire 3D TRUS volume and a volume-volume registration may be performed. ${ }^{5,6}$ However, neither $3 \mathrm{D}$ TRUS probe is commonly available in diagnostic centers nor the use of the EM tracker is an established clinical practice except for research validations. Therefore, intending to solve the 2D TRUS-MR slice correspondence problem, we propose a method based on Chi-square distance of shape-context representations of the prostate contours and information theoretic measure of Normalized Mutual Information (NMI) of the TRUS-MR slices. Finally, a strategic elimination method between the Chi-square distances and NMI measure is used to choose the MR slice among a set of slices which is the closest to the TRUS slice. The novelties of the proposed work may be summarized as follows:

1. Using shape context representations of the contours to find prostate shape similarities between TRUS and MR slices.

2. An elimination method that exploits shape information (here shape context) and intensity information (NMI) to identify the correct MR slice.

In the remaining paper, section 2 describes the proposed method in detail, section 3 provides the results and discussions followed by the conclusions in section 4 .

\section{THE PROPOSED METHOD}

In this work, the prostate is manually segmented from the 2D TRUS axial slice and the pre-biopsy axial MR volume (base to apex slices). Manual segmentation ensures that automatic segmentation errors are not incorporated in the evaluation process. However, we are also investigating on automatic prostate segmentation methods in both MR and US modalities. ${ }^{7,8}$ The shape similarity measure using Chi-square distance is explained in section 2.1, the information theoretic image similarity measure of NMI is explained in section 2.2 with an explanation of the strategic elimination approach in section 2.3 .

\subsection{Shape Similarity}

The segmented prostate contour points are uniformly sampled using fixed Euclidean distance of $\varepsilon$ i.e. if $c_{i}$ is a contour point, $i=1, \ldots, N$, then maximize the following equation

$$
\underset{j}{\arg \max }\left\|c_{i}-c_{j}\right\|^{2} \leq \varepsilon, \quad i \neq j .
$$

Figure 1 shows the uniformly sampled segmented contours on the TRUS and MR images. Let the number of uniformly sampled points now be represented as $n$, then each sample point $c_{i}$ may be represented by a shape descriptor that is a $n-1$ length vector of log-polar relative distances to points $c_{j}$, where $i \neq j$. The shape descriptor is binned into a histogram that is uniform in log-polar space and this histogram is the shape-context representation of a contour point ${ }^{9}$ i.e. $c_{i}$ is represented by a histogram $h_{i}(k, \theta)$ such that

$$
h_{i}(k, \theta)=\#\left\{c_{j}, i \neq j \quad: \quad\left(c_{i}-c_{j}\right) \in \operatorname{bin}(k, \theta)\right\} .
$$




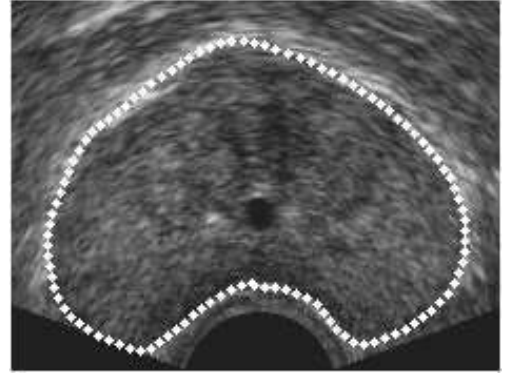

(a)

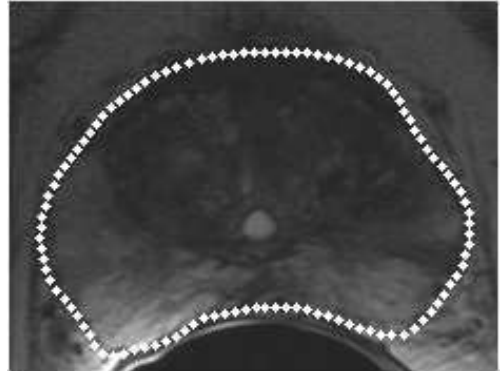

(b)

Figure 1. Uniformly sampled contours. (a) and (b) are the uniformly sampled segmented prostate contours on the TRUS and MR images respectively.

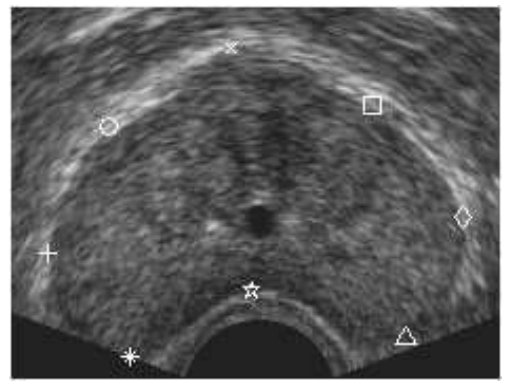

(a)

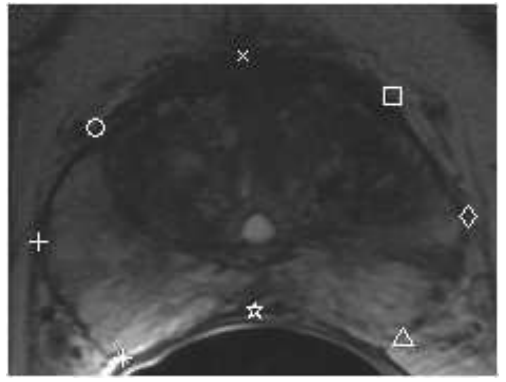

(b)

Figure 2. Point correspondences example. (a) contour points in TRUS, (b) point correspondences of (a) in MR.

$k$ is the $\log r=\log \left(\sqrt{\left(x_{i 1}-x_{j 1}\right)^{2}+\left(x_{i 2}-x_{j 2}\right)^{2}}\right)$ and $\theta=\tan ^{-1} \frac{x_{j 2}-x_{i 2}}{x_{j 1}-x_{i 1}}$ of the relative distance $\left(c_{i}-c_{j}\right)$, where, $c_{i}=\left(x_{i 1}, x_{i 2}\right)$ and $c_{j}=\left(x_{j 1}, x_{j 2}\right)$. As suggested by Belongie et al., ${ }^{9}$ a total of 5 bins are considered for $k$ and 12 bins for $\theta$ that ensures that the histogram is uniform in log-polar space. This also means that more emphasis is given to the nearby sample points than those that are far away.

In the original work of Belongie et al. ${ }^{9}$ the point correspondence between two shapes is obtained by a

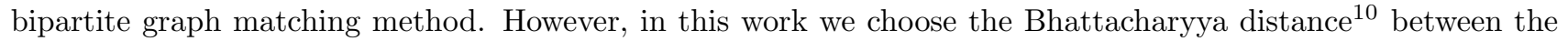
shape-context histograms of two shapes to find the best point correspondence since it is fast to compute and statistically a robust measure to find correspondences in similar shapes like prostate contours in TRUS and MRI. Thus, to match a point $c_{i}$ in a shape to a point $c_{j}^{\prime}$ in another shape, the Bhattacharyya coefficients between the shape-context histogram of $c_{i}$ and all $c_{j}^{\prime}$ are computed and the $c_{j}^{\prime}$ that maximizes the relation in Eq. (3) is chosen as the corresponding point.

$$
\underset{c_{j}^{\prime}}{\arg \max } \sum_{k=1}^{5} \sum_{\theta=1}^{12} \sqrt{\hat{h}_{i}(k, \theta) \cdot \hat{h}_{j}^{\prime}(k, \theta)}
$$

where, $\hat{h}_{i}(k, \theta)$ and $\hat{h}_{j}^{\prime}(k, \theta)$ are the normalized shape-context histograms of $c_{i}$ and $c_{j}^{\prime}$ respectively. Fig. 2 shows the contour correspondences overlaid on the TRUS and MR prostate shapes.

After the corresponding points are identified, the Chi-square $\left(\chi^{2}\right)$ distances between the TRUS slice and each of the MR slices are calculated based on the corresponding shape-context histograms and is given by $\mathcal{C}_{i j}$ in Eq. (4). The final distance is the sum of all the $\chi^{2}$ distances of the corresponding points (shape-context histograms) in TRUS and MR and is given by $\mathcal{H}$ in the following equation.

$$
\mathcal{C}_{i j}=\frac{1}{2} \sum_{k=1}^{5} \sum_{\theta=1}^{12} \frac{\left(\hat{h}_{i}(k, \theta)-\hat{h}_{j}^{\prime}(k, \theta)\right)^{2}}{\hat{h}_{i}(k, \theta)+\hat{h}_{j}^{\prime}(k, \theta)}, \quad \mathcal{H}=\sum_{i=1}^{l} \mathcal{C}_{i j},
$$


where, $l$ is the number of point correspondences. The TRUS-MR slice pair with minimum sum of $\chi^{2}$ distance $(\mathcal{H})$ is identified and its significance will be discussed in the following subsection.

\subsection{Image Similarity}

Image similarity measures have been extensively used in multimodal image registration problem to ensure that the moving image is transformed with close resemblance to the fixed image. In this work, our problem is to find an MR slice in the volume that closely resembles the TRUS slice. Therefore, to find such similarity we employ the well-known NMI as the image similarity measure.

The TRUS-MR slice pair identified with the minimum $\mathcal{H}$ as obtained from Eq. (4) is used to retrieve the $2 \mathrm{D}$ rigid transformation (inplane rotation and translation) parameters between them; and the remaining MR slices in the volume are rigidly registered with the TRUS slice using the same parameters. This registration step ensures similar 2D inplane rigid alignment of all the MR slices of the volume with the 2D TRUS slice.

After the alignment of the MR volume with the TRUS slice, pairwise NMI are computed for each MR-TRUS slice pair. The NMI is an information theoretic measure that tries to reduce the joint entropy of the images ${ }^{11}$ and is given by

$$
\mathrm{NMI}=\frac{H(M)+H(T)}{H(M, T)}
$$

where, $H(M)$ and $H(T)$ are the marginal entropies of the MR $(M)$ and TRUS $(T)$ images respectively, and $H(M, T)$ is the joint entropy of the images. $H(M, T)$ can be written using probability theory as

$$
H(M, T)=-\sum_{M, T} p(M, T) \log [p(M, T)],
$$

where, $p(M, T)$ is the joint probability distribution of the images obtained from their joint histogram.

\subsection{Slice Selection}

Intuitively, the MR slice that has the maximum overlap with the TRUS slice and similar internal gland structures will have the maximized NMI as obtained from Eq. (5), and may be identified as the corresponding MR slice. However, such straightforward conclusions do not lead to the MR slice that spatially corresponds to the TRUS slice. Therefore, we propose a rule-based elimination process considering both the sum of $\chi^{2}$ distances $(\mathcal{H})$ and the NMI.

A set of two rules are designed to eliminate the slices from a set of potential candidate slices. The potential candidate slices are the ones with distances less than the mean of the $\mathcal{H}$ distances. The resulting set is definitely a group of consecutive MR slices. From this set, we identify the ones with lowest NMI and lowest $\mathcal{H}$ distance, if both coincide at the same slice, we identify that MR slice as the corresponding one to that of the TRUS slice. Otherwise, we use one of the following rules (i-ii), based on a condition.

Condition: Check if the $\mathcal{H}$ distance of the slice corresponding to the lowest NMI $\leq$ lowest $\mathcal{H}$ distance + standard deviation of $\mathcal{H}$ distances.

Rules:

1. If the condition is FALSE then choose the slice with lowest $\mathcal{H}$ (sum of Chi-square) distance.

2. If the condition is TRUE then choose the slice with lowest NMI.

\section{RESULTS AND DISCUSSIONS}

The proposed method has been validated against the ground truth obtained from one expert radiologist and one expert urologist for 15 patients. Axial mid-gland TRUS slices were available to validate the results. Table 1 shows the ground truth provided by the experts (independently) and the results we obtained using our method. It is observed from the table that our results matched at least one expert for 11 out of 15 patients wherein the experts differ among their opinions by one slice in 4 cases i.e. for patients $1,4,8,9$, and in 6 patient cases i.e. 
Table 1. Ground truth (GT) and results for MR slices corresponding to a TRUS slice.

\begin{tabular}{|c|c|c|c|c|c|c|c|c|c|c|c|c|c|c|c|c|}
\hline Patients/MR Slice & 1 & 2 & 3 & 4 & 5 & 6 & 7 & 8 & 9 & 10 & 11 & 12 & 13 & 14 & 15 & Agreement (\%) \\
\hline GT (Expert 1) & 8 & 7 & 6 & 10 & 6 & 10 & 5 & 7 & 12 & 8 & 6 & 5 & 7 & 6 & 7 & $\mathbf{6 6 . 6 7}$ \\
\hline GT (Expert 2) & 7 & 5 & 6 & 9 & 6 & 8 & 7 & 6 & 13 & 8 & 4 & 8 & 10 & 6 & 7 & $\mathbf{1 0 0}$ \\
\hline Our method & 7 & 6 & 6 & 9 & 6 & 8 & 8 & 6 & 13 & 7 & 4 & 8 & 10 & 6 & 6 & - \\
\hline
\end{tabular}

$2,6,7,11,12,13$ their opinions differ by $2-3$ slices. The expert results matched in 5 cases (patients $3,5,10,14$ and 15) out of which our results agreed with 3 patients cases (3,5 and 14), while only one slice away for the remaining 2 cases. Our results do not match with any expert for the patient cases 2,7,10 and 15. In such cases where the experts disagreed on their opinions, our results are just one slice away from either or both the ground truth. The matched cases with one or both the expert ground truth are shown in italics in the table.

Comparing each of the expert ground truth independently with our method, the exact matches with expert 1 ground truth are for 3 cases (patients 3,5 and 14) and one slice away matches are for 7 cases (patients $1,2,4,8,9,10$ and 15 respectively). Therefore, 10 out of 15 cases i.e. $66.67 \%$ results are in agreement with that of the ground truth of expert 1 . Similarly, for 11 cases, the results of our method are in exact agreement with expert 2 ground truth, while one slice away for the remaining 4 cases (patients 2, 7, 10 and 15). This signifies that the results of our method are in $100 \%$ agreement with that of expert 2 ground truth. Therefore, we may conclude that in all cases our results are close enough to the ground truth if not exactly matched with either or both of them.

Fig. 3 shows the patient cases 1, 4, 8 and 9 where the results of our method are in agreement with expert 2 ground truth but one slice away from expert 1 ground truth. It may be observed from the figure that the expert ground truth being one slice away from one another, have almost similar internal gland structures for the MRI slice. Similarly, Fig. 4 shows those patient cases 10 and 15 where our results are one slice away from both the experts ground truth. Fig. 5 shows the two patient cases 2 and 7 where our results matched none of the experts ground truth. Fig. 5 reveals that the choice of the MR slice using our method is almost similar to that of the experts ground truth.

The method has been implemented in MATLAB and the complete process takes 3 secs on an average to find out the corresponding MR slice from a set of $12-14$ slices. It is to be noted that $\mathrm{Xu}$ et al. ${ }^{4}$ employed an EM tracker to locate the spatial position of the 2D TRUS slice (during guided biopsy) in the 3D TRUS volume. Thereafter, to compensate for the prostate motion, the sum-of-squared difference (SSD) between the maximum translational and rotational TRUS slices within a short time frame (during TRUS guided biopsy) and the corresponding spatial 2D TRUS slices obtained in the 3D TRUS volume was minimized. However, in our case the exact orientation of the 2D TRUS probe relative to the prostate is unknown. Therefore, we may similarly propose the minimization of SSD or NMI between the 2D MR slice corresponding to the 2D TRUS slice (obtained from our method) and the corresponding MR slices of the maximum translational and rotational TRUS slices during biopsy. Hence, our proposed method provides a good starting point for multimodal registration and may be used as a substitute of EM tracker and 3D TRUS probe that are generally not used for biopsy.

\section{CONCLUSIONS AND FUTURE WORKS}

A method to find out 2D MR slice correspondence of a 2D axial TRUS slice during biopsy has been reported in this paper. The method is based on shape-context and statistical measures and is fast in finding out correspondences that are nearly the same as the ground truth obtained from two experts. Since EM tracker is not easily available in hospitals in Europe and 3D TRUS is normally not employed for biopsy purposes, our proposed method may provide a good starting point for multimodal fusion of TRUS-MR images to improve the sampling of biopsy tissues. Although the results reported in this paper are validated only for mid-gland or close to mid-gland axial slices, the validations for the base and apex TRUS axial slices and TRUS sagittal slices and cross-validation of our method with the use of EM tracker have been left as future works. 


\section{ACKNOWLEDGMENTS}

This research is a part of the PROSCAN project of the VICOROB laboratory of University of Girona, Catalunya, Spain. The authors would like to thank VALTEC 08-1-0039 of Generalitat de Catalunya, Spain and Conseil Régional de Bourgogne, France for funding this research.

\section{REFERENCES}

[1] Carroll, P. and Shinohara, K., "Transrectal ultrasound guided prostate biopsy," tech. rep., Department of Urology, University of California, San Francisco (2010). http://urology.ucsf.edu/patientGuides.html, accessed [30th Dec, 2010].

[2] Arcangeli, C. G., Ornstein, D. K., Keetch, D. W., and Andriole, G. L., "Prostate-specific antigen as a screening test for prostate cancer-The United States experience," The Urologic Clinics of North America 24(2), 299-306 (1997).

[3] Vilanova, J. C., Barceló-Vidal, C., Comet, J., Boada, M., Barceló, J., Ferrer, J., and Albanell, J., "Usefulness of prebiopsy multi-functional and morphologic MRI combined with the free-to-total PSA ratio in the detection of prostate cancer," American Journal of Roentgenology 196(6), W715-W722 (2011).

[4] Xu, S., Kruecker, J., Turkbey, B., Glossop, N., Singh, A. K., Choyke, P., Pinto, P., and Wood, B. J., "Real-time MRI-TRUS fusion for guidance of targeted prostate biopsies," Computer Aided Surgery 13(5), $255-264$ (2008).

[5] Baumann, M., Mozer, P., Daanen, V., and Troccaz, J., "Prostate biopsy assistance system with gland deformation estimation for enhanced precision," in [Proc. of MICCAI], LNCS 5761, 57-64 (2009).

[6] Baumann, M., Mozer, P., Daanen, V., and Troccaz, J., "Prostate biopsy tracking with deformation estimation," Medical Image Analysis, In Press (2011).

[7] Ghose, S., Oliver, A., Martí, R., Lladó, X., Freixenet, J., Vilanova, J., and Meriaudeau, F., "Prostate segmentation with texture enhanced active appearance model," in [Proc. IEEE International Conference on Signal-Image Technology 83 Internet-Based Systems], 18-22 (December 2010).

[8] Ghose, S., Oliver, A., Martí, R., Lladó, X., Freixenet, J., Vilanova, J., and Meriaudeau, F., "A probabilistic framework for automatic prostate segmentation with a statistical model of shape and appearance," in [Proc. IEEE International Conference on Image Processing], 725-728 (September 2011).

[9] Belongie, S., Malik, J., and Puzicha, J., "Shape matching and object recognition using shape contexts," IEEE Transactions on Pattern Analysis and Machine Intelligence 24, 509-522 (April 2002).

[10] Bhattacharyya, A., "On a measure of divergence between two statistical populations defined by their probability distribution," Bulletin of the Calcutta Mathematical Society 35, 99-110 (1943).

[11] Studholme, C., Hill, D. L. G., and Hawkes, D. J., "An overlap invariant entropy measure of 3D medical image alignment," Pattern Recognition 72(1), 71-86 (1999). 

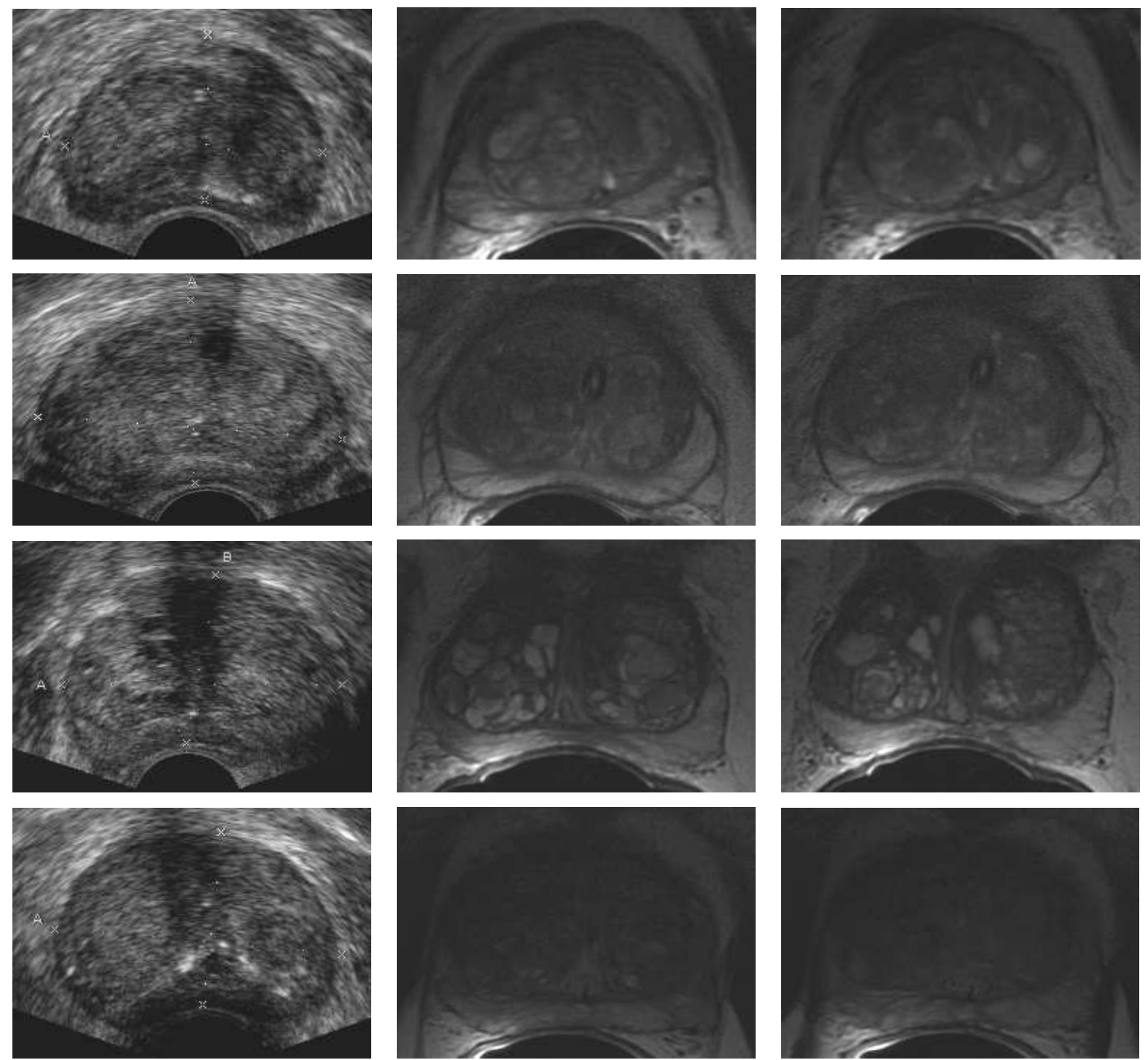

Figure 3. Results using our method that match expert 2 ground truth and one slice away from expert 1 ground truth. The rows show the results for the patients $1,4,8$ and 9 . The first column shows the US slice and columns two and three show the respective ground truth MR slices of expert 1 and 2 respectively. 

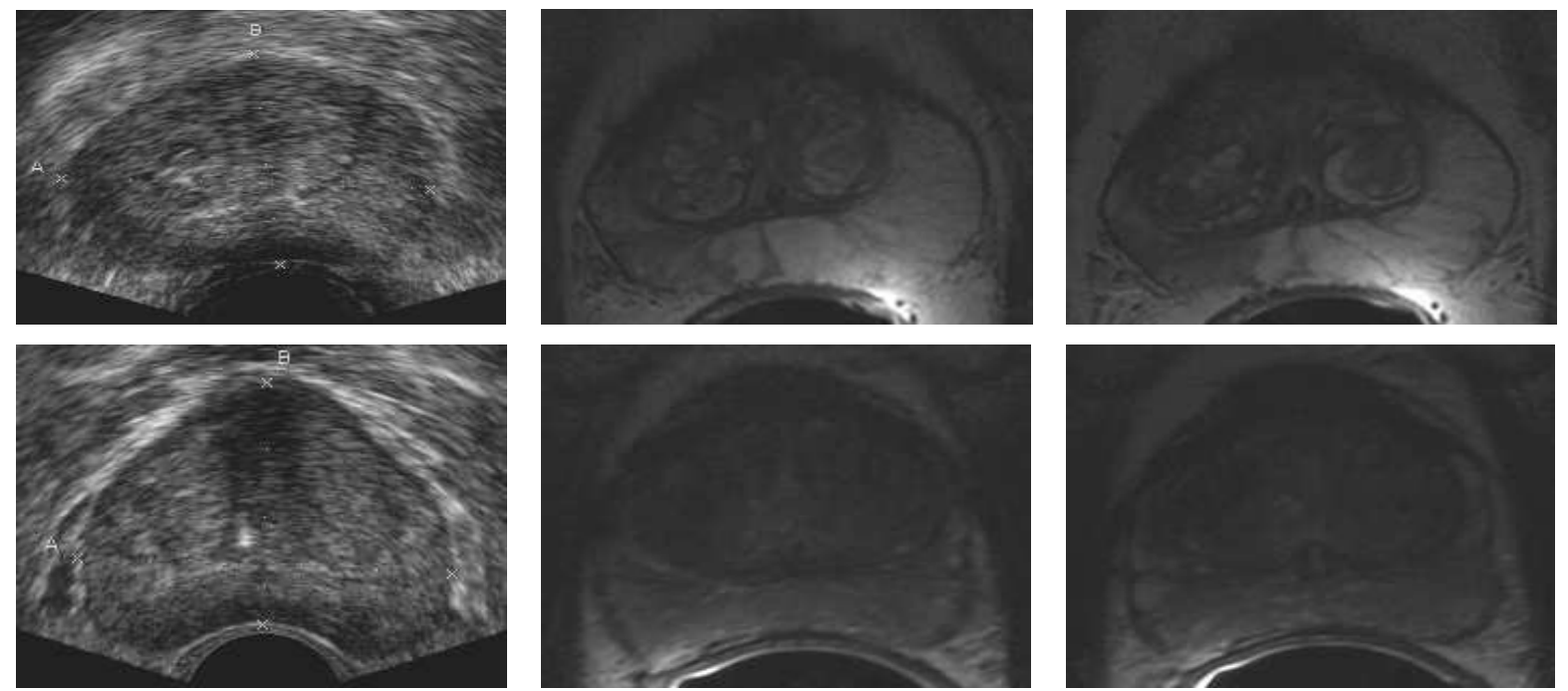

Figure 4. Results using our method that are one slice away from expert $1 \& 2$ ground truth. The rows show the results for the patients 10 and 15. The first column shows the US slice, column two shows the experts choice (both expert $1 \&$ 2 ) and the third column shows the MR slice obtained using our method.
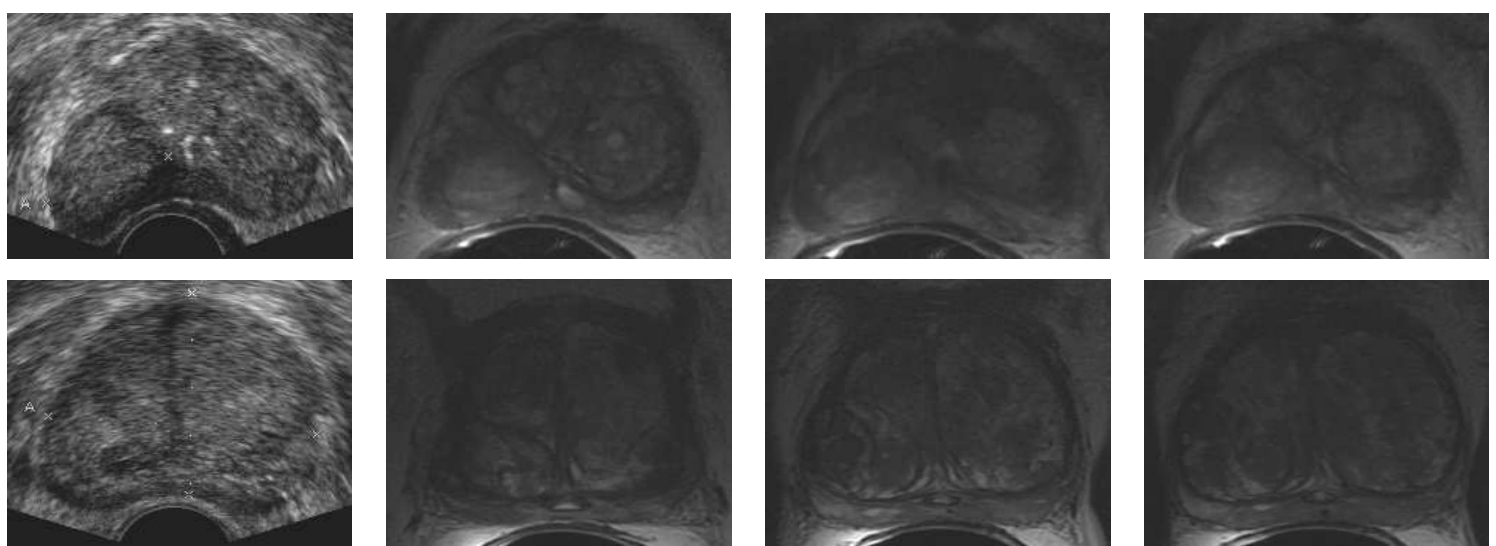

Figure 5. Results using our method that do not match any of the expert ground truth. The rows show the results for the patients 2 and 7. The first column shows the US slice, column two and three show the expert $1 \& 2$ ground truth respectively and the fourth column shows the MR slice obtained using our method. 